\section{Core formation and Earth's late accretionary history}

A RECENT paper by Morgan' concerning the origin of refractory noble siderophile elements in the Earth's upper mantle contains statements which may be misleading to interested readers who have not followed in detail the recent debates on core formation and mantle siderophile elements. Morgan used the approximately chondritic Os/Re ratio inferred for the Earth's Mantle from the Os isotopic systematics of terrestrial osmiridiums to examine three hypotheses of core formation: (1) heterogeneous accretion/"chondritic veneer'2; (2) equilibrium between mantle silicates and a eutectic $\mathrm{Fe}-\mathrm{S}-\mathrm{O}$ liquid $^{3}$; and (3) inefficient core formation ${ }^{4}$. Morgan concludes on the basis of both terrestrial and meteoritic Os/ $\mathrm{Re}$ ratios that the heterogeneous accretion/chondritic veneer (or 'late influx') hypothesis is most likely and should be favoured.

We feel that two points of Morgan's paper deserve further discussion. First, as regards our own model of inefficient core formation, even very small amounts of trapped metal will tend to buffer the residual mantle at the $\mathrm{Os} / \mathrm{Re}$ ratio which existed before core formation. Secondly, as a consequence of the first point, it is unclear to us whether or not the $\mathrm{Os} / \mathrm{Re}$ ratio of the present mantle has been modified, relative to the bulk Earth value, by mantle processes such as core formation or extraction of basaltic magma. In the absence of knowledge of the bulk Earth Os/Re ratio, it is not obvious how one establishes that the present $\mathrm{Os} / \mathrm{Re}$ ratio of the upper mantle reflects the addition of a chondritic 'late-stage veneer', rather than resulting from in situ processes.

We disagree with Morgan's evaluation of hypothesis (3). The hypothesis postulates that the approximately chondritic ratios of the refractory noble siderophile elements in the mantle result from the retention of very small amounts of solid metal and S-bearing metallic liquid during segregation of the core from the mantle (that is, core formation was not $100 \%$ efficient ${ }^{4}$ ), with subsequent oxidation of that metal. Morgan ${ }^{1}$ states that chondritic ratios of refractory noble siderophile elements are not a natural consequence of this hypothesis. This statement is correct as a generality because fractionations between noble siderophile elements are, in principle, allowed during geologic processes. However, in practice, Morgan's statement is not correct as applied to our model of inefficient core formation because trace amounts of trapped solid metal will tend to buffer the mantle towards the noble metal abundance ratios which existed before core formation. The (solid metal/silicate liquid) and (liquid metal/silicate liquid) partition coefficients for Os (by analogy with Ir) are $\sim 10^{6}$ and $\sim 10^{4}$, respectively, while those for $\mathrm{Re}$ are $\geqslant 10^{5}$ and $\geqslant 10^{3}$, respectively ${ }^{5}$. Thus, if core formation is not completely efficient, trace amounts of trapped solid metal will tend to dominate the other Os- $\mathrm{Re}$ reservoirs in the mantle, closely preserving original noble metal abundance ratios in the sub. sequently oxidized upper mantle.

For example, model calculations indicate that the fractionation of the mantle $\mathrm{Os} / \mathrm{Re}$ ratio relative to the bulk Earth value during inefficient core formation is less than the range of $\mathrm{Os} / \mathrm{Re}$ ratios observed in chondritic meteorites. Because Os behaves similarly to Ir in geochemical processes such as core formation $^{6}$ and peridotite partial melting ${ }^{7}$, Os may be preferentially incorporated into the Earth's core relative to $\mathrm{Re}$, resulting in a mantle with a slightly lower Os/Re ratio than the bulk Earth. However, even if the (solid metal/liquid metal), (liquid metal/liquid silicate) and (solid silicate/liquid silicate) partition coefficients for Os are larger than those for $\mathrm{Re}$ by factors of 2,10 and 50, respectively, the $\mathrm{Os} / \mathrm{Re}$ ratio in the mantle will typically deviate by less than $30 \%$ from the original $\mathrm{Os} / \mathrm{Re}$ ratio-less than the range of the means of the chondrite groups reported by Morgan ${ }^{1}$.

This calculation is most sensitive to the relative values of the (solid metal/liquid metal) partition coefficients chosen for Os and $\mathrm{Re}$. The relative constancy of $\mathrm{Os} / \mathrm{Re}$ and $\mathrm{Os} / \mathrm{Ir}$ ratios in magmatic iron meteorite groups, whose $\mathrm{Os}, \mathrm{Re}$ and $\mathrm{Ir}$ concentrations change by up to four orders of magnitude ${ }^{6}$, argues that Os, Re and Ir behave rather coherently during core formation and solidification processes. This observation suggests that the factor of two difference which we have used in our sample calculation represents a firm limit on the allowable difference between Os and Re. Of course, if the partition coefficients for Os and Re were identical, no fractionation would occur in metallic systems and the $\mathrm{Os} / \mathrm{Re}$ ratio of the mantle would remain essentially unchanged during inefficient core formation.

As shown in Morgan's ${ }^{1}$ Fig. 1, the Os/ $\operatorname{Re}$ weight ratio in individual samples of 12 types of chondritic material varies from $\sim 8$ to $\sim 19$ (a factor of 2.4 ), although average values for chondrite groups (which are probably less sensitive to sampling) exhibit a smaller range, from $\sim 8$ to 14. Evidently there is no exact value of the 'chondritic' Os/ $\operatorname{Re}$ ratio and, in the absence of convincing independent evidence that the Earth is made of a particular type of chondrite material, it is not possible to determine that the mantle $\mathrm{Os} / \mathrm{Re}$ ratio has been unaffected by metal/silicate segregation. For example, if the initial bulk Earth Os/Re ratio were identical to that of CI chondrites $(\mathrm{Os} / \mathrm{Re}=14$; ref. 8$)$, a $15 \%$ reduction could change the mantle $\mathrm{Os} / \mathrm{Re}$ ratio to 12 - a value consistent with isotopic and chemical data obtained from mantlederived materials ${ }^{1,9}$, and consistent with our upper limit on the likely change in the mantle Os/Re ratio during core formation. (We note that, in an earlier version of this comment, we took the $\mathrm{Os} / \mathrm{Re}$ ratio of $\mathrm{CI}$ chondrites to be 19-a mistake caused by an error in the abundance of Os in Orgueil given in Table 1 of ref. 8 . The interested reader should change this figure from 699 to 504 p.p.b. in ref. 8 (E. Anders, personal communication). We thank J. W. Morgan for catching this error of calculation and pointing it out to us.) Thus, because both the exact value of the Earth's Os/Re ratio and the exact amount of fractionation of Os from $\mathrm{Re}$ during core formation are unknown, it does not seem possible to regard the mantle $\mathrm{Os} / \mathrm{Re}$ ratio as pristine simply because the mantle value falls within the chondritic range.

We conclude that $\mathrm{Os} / \mathrm{Re}$ fractionation between mantle and core is only significant when core formation is efficient and no solid metal ( $\leqslant 0.04 w t \%)$ is retained by the mantle. Although minor Os/Re fractionations are possible, their magnitudes are typically less than the range of the mean Os/ Re ratios of individual chondrite groups. Even though the Earth's mantle has an $\mathrm{Os} / \mathrm{Re}$ ratio within the range observed in chondritic materials, that value could still have been altered by coreforming processes and is not necessarily primordial. Ratios of moderately siderophile elements such as $\mathrm{Co} / \mathrm{Ni}$ do pose problems for inefficient core formation models ${ }^{5}$, but ratios of refractory noble siderophile elements such as $\mathrm{Os} / \mathrm{Re}$ do not. Of all the evidence for a late-stage chondritic veneer, the $\mathrm{Os} / \mathrm{Re}$ ratio of the Earth's mantle is, in our view, the least conclusive.

We would like to thank John Morgan for a scholarly, friendly exchange of views, and Ed Anders for clarifying cosmic Os abundances. This work was supported by NASA grant NAG 9-39.

JOHN H. JONES

MichaEl J. DRAKE

Lunar and Planetary Laboratory, University of Arizona, Tucson, Arizona 85721, USA

1. Morgan, J. W. Nature 317, 703-705 (1985).

Wänke, H. Phil Trans. R. Soc. A303, 289-302 (1981)

Brett, R. Geochim. cosmochim. Acta 48, 1183-1188 (1984). 4. Jones, J. H. \& Drake, M. J. Lunar planet. Sci. XV, 1183-1188 (1984)

5. Jones, J. H. \& Drake, M. J. Nature 322, 221-228 (1986). 6. Scott, E. R. D. Geochim. cosmochim. Acta 36, 1205-1236 (1972).

7. Chou, C.-L., Shaw, D. M. \& Crockett, J. H. J. geophys. Res. 88, A507-A518 (1983).

8. Anders, E. \& Ebihara, M. Geochim. cosmochim. Acta 46, 2363-2380 (1982).

9. Luck, J. M. \& Allègre, C. J. Nature 283, 130-132 (1983).

MORGAN REPLIES-My letter ${ }^{1}$ raises two major points. First, the chondritic Os/Re 